\title{
Fragment de description de la physique des transferts thermiques dans le sous-sol
}

\section{Fragment of Description of Heat Transfer Physics in the subsoil}

\author{
Serge Bories \\ Institut de Mécanique des Fluides de Toulouse \\ Laboratoire Associé au C.N.R.S. \\ 2, rue Charles Camichel, 31071 Toulouse Cedex
}

\begin{abstract}
Malgré une parfaite identification et une bonne connaissance des phénomènes physiques élémentaires, l'élaboration de modèles conceptuels rigoureux, permettant de décrire les transferts thermiques dans le sol, se heurte à de nombreuses difficultés liées à la nature essentiellement hétérogène du milieu.

Lorsque certaines de ces difficultés peuvent être levées, par recours au concept de milieu poreux dit homogène, la prédétermination des transferts thermiques est rendue possible grâce à un formalisme théorique, dont la validité a été appréciée par des expériences de laboratoire. Cette modélisation peut servir de base d'analyse et d'interprétation de certains phénomènes de transfert se développant dans le sous-sol.
\end{abstract}

Although the elementary physical phenomena are fully identified and well known, there are many difficulties in establishing strict conceptual models to describe heat transfers in the soil, mainly related to the heterogeneous nature of the medium.

When some of these difficulties can be overcome by recourse to the concept of porous medium, said to be homogeneous, the heat transfers can be predetermined by theoretical formalism, whose validity was demonstrated by laboratory experiments. This model can be used as basis for analyzing and interpreting certain transfer phenomena which occur in the subsoil.

\section{Introduction}

L'importance croissante prise par les dépenses énergétiques dans les économies des pays industrialisés a conduit, au cours de la dernière décennie, à la mise en place de programmes de recherche sur le développement de nouvelles technologies et l'exploitation de certaines ressources jusqu'alors négligées.

Dans ce contexte, l'utilisation du sous-sol pour la mise en œuvre de solutions visant à fournir des calories à basse et moyenne température, destinées au chauffage domestique et parfois même à la production d'électricité, est apparue comme une orientation sinon privilégiée, tout au moins importante. Outre les gisements géothermiques naturels, constitués par les aquifères, c'est en effet l'ensemble du sous-sol qui, grâce à divers procédés fondés sur le principe des échangeurs, fait désormais l'objet d'études en vue d'une exploitation thermique.

Quel que soit le type de gisement exploité et le mode de récupération des calories envisagé, le dimensionnement des équipements, la durée d'exploitation et en particulier la gestion optimale de la ressource ou du stockage, ne peuvent être précisés que si l'on dispose d'une bonne connaissance des transferts de chaleur et des transferts de masse qui leur sont bien souvent associés et qui contrôlent la cinétique du transfert d'énergie dans les formations géologiques exploitées. Pour une large part, l'étude de ces phénomènes fait appel à la physique des transferts dans les milieux poreux, domaine dans lequel, comme nous le montrerons à travers quelques exemples, des progrès significatifs ont été réalisés au cours de la dernière décennie.

Même si, en raison de la diversité et de la complexité des situations géologiques naturelles, les connaissances acquises en la matière s'avèrent encore parfois insuffisantes dans une perspective d'exploitation et de gestion entièrement déterministe des gisements, elles n'en demeurent pas moins pour l'instant le seul recours possible dans l'analyse et l'interprétation rationnelle des mécanismes mis en jeu. 


\section{Rappels sur les fondements de la modélisation mathématique en milieu poreux}

On désigne communément par milieu poreux un solide rigide de forme compliquée englobant des vides appelés pores. Ces vides peuvent communiquer entre eux et contenir une ou plusieurs phases fluides susceptibles de s'écouler et éventuellement d'échanger entre elles et/ou avec le solide de la matière et de l'énergie.

Lorsque les dimensions géométriques des pores et des fractions de la phase solide comprises entre pores, ont des valeurs largement supérieures aux dimensions moléculaires, chaque phase peut être considérée comme un milieu continu et le milieux poreux comme un ensemble de milieux continus étroitement imbriqués. Dans cette acception, les phénomènes qui se déroulent au sein du milieu poreux (écoulements et phénomènes de transferts) sont parfaitement décrits par les équations dites microscopiques de la thermodynamique des milieux continus et la détermination du champ des différentes variables en tout point de chacune des phases devrait, a priori, pouvoir être effectué.

En raison de la complexité géométrique de l'espace poreux et des difficultés, pour l'instant insurmontables, que pose la définition précise des domaines occupés par chacune des phases, la description microscopique, dont nous venons de parler, ne peut toutefois être mise en pratique, qu'après un changement d'échelle dont l'étape essentielle conduit à définir un Volume Elémentaire Représentatif (V.E.R.), permettant d'établir une équivalence entre le milieu dispersé réel et un milieu continu fictif. Par opposition avec l'échelle microscopique, telle qu'elle est proposée en mécanique des milieux continus, l'échelle du milieu continu fictif équivalent, définie à partir d'un élément de volume poreux dont les dimensions sont grandes auprès des dimensions des pores, est dite macroscopique. Dans cette nouvelle échelle, les phénomènes sont décrits en moyenne par des équations dont les variables et les paramètres sont représentatifs des variables et paramètres physiques moyens au sein du continuum de milieu poreux.

Bien qu'il existe, à l'heure actuelle, des tentatives de modélisation à l'échelle macroscopique fondées sur le calcul en moyenne des équations microscopiques écrites pour chacune des phases, nous verrons ultérieurement que les difficultés rencontrées tant au point de vue physique que mathématique sont encore loin d'être surmontées et qu'en pratique on est conduit à faire un certain nombre d'hypothèses que seule l'expérience permet de justifier a postériori.

\subsection{Concept de Volume Elémentaire Représentatif - Porosité}

Lorsque l'on aborde l'étude des écoulements en milieu poreux, deux échelles s'imposent au choix de l'observateur pour analyser les phénomènes :
- l'échelle du pore ou des fissures, à l'intérieur de laquelle les grandeurs locales ou microscopiques peuvent largement varier,

- l'échelle du milieu poreux, ou macroscopique, caractéristique de variations significatives de ces mêmes grandeurs, définies en moyenne, sur un certain volume de milieu poreux.

En règle générale, l'échelle microscopique est associée au diamètre moyen des pores, $d$, et l'échelle macroscopique $L$, à une dimension géométrique du milieu.

Pour définir le concept de V.E.R., appelons $\psi_{a}$ une grandeur (vitesse, masse volumique, ...) associée à la phase $\alpha$ définie dans l'espace des pores en tout point du milieu et soit :

$$
\left\langle\psi_{a}\right\rangle=\frac{1}{R} \int_{R} \psi_{a} \mathrm{~d} V
$$

la moyenne volumique de $\psi_{a}$ dans un volume $R$ centré en un point repéré par le vecteur de position $\vec{x}$ (Fig. 1). En général $\left\langle\psi_{a}\right\rangle$, sera fonction du volume, de la forme et de l'orientation de $R \overrightarrow{(\vec{x})}$ au temps $t$. Pour que $\left\langle\psi_{a}\right\rangle$ fonction continue soit indépendante de ces facteurs et ne dépende que de $\vec{x}$ et de $t$, il convient de sélectionner un volume $R_{0}$ tel que $\left\langle\psi_{a}\right\rangle$ soit stationnaire par rapport à $\boldsymbol{R}_{0}$, soit : $\partial\left\langle\psi_{a}\right\rangle / \partial \boldsymbol{R}_{0}=0$. Si une telle condition peut être dégagée quel que soit $\vec{x}$ dans un certain domaine du milieu poreux, ce dernier pourra être considéré comme macroscopiquement continu pour $\left\langle\psi_{a}\right\rangle \cdot R_{0}$ l'échelle de continuité de $\left\langle\psi_{a}\right\rangle$ est appelé V.E.R. Un système multiphasique $\alpha=1,2, \ldots, n$, pourra être traité comme continu pour la grandeur $\psi_{a}$ si l'échelle de continuité $R_{0}$ est commune à l'ensemble des phases.

Pour illustrer les conditions auxquelles doit satisfaire $R_{0}$, considérons un point mathématique $P$ situé dans un domaine poreux et examinons l'évolution de

$$
\left\langle\psi_{a}\right\rangle=\frac{1}{R} \int_{R} \psi_{a} \mathrm{~d} V
$$

définie sur $R$ centré en $P$ lorsque $R$ varie. $\left\langle\psi_{a}\right\rangle$ prend les valeurs 0 ou $\psi_{a}$ (valeur locale) (Fig. 2), selon que $P$ se trouve dans la phase considérée ou non lorsque $R \rightarrow 0$, puis tend à évoluer sans fluctuation notable lorsque $R>R_{0}$. Les fluctuations qui apparaissent lorsque $R<R_{0}$ résultant de l'influence discrète de la distribution de la phase à l'échelle microscopique (pore) lors de la sommation effectuée sur $R$, la première restriction que nous imposerons intuitivement aux dimensions, 1 , de $R_{0}$, sera donc : $1>d$. Cette condition permettant d'assurer le "lissage " de $\left\langle\psi_{a}\right\rangle$ en limitant l'incidence de la distribution aléatoire de la phase $\alpha$ liée à l'espace des pores, il convient de rechercher maintenant à quelle condition $\left\langle\psi_{a}\right\rangle$ définie sur $R_{0}$ (macropoint $P$ ), mais en règle générale variable avec $R$ sur l'échelle $L$ du milieu poreux, reste représentative pour tout point $P^{\prime}$ situé dans $R_{0}$ centré en $P$. Si $\left\langle\psi_{a}\right\rangle_{P}$, représente la valeur moyenne de $\psi_{a}$ 
sur un élément de volume $R_{0}$ centré en $P^{\prime}$ avec $P^{\prime} \in\left(R_{0}\right.$ centré en $P),\left\langle\psi_{a}\right\rangle$ sera bien représentative de tous les points $P^{\prime}$ contenus dans $R_{0}$ centré en $P$ (Fig. 1 ) dès lors que :

$$
\left\langle\left\langle\psi_{a}\right\rangle_{P^{\prime}}\right\rangle=\frac{1}{R_{0}} \int_{R_{0}}\left\langle\psi_{a}\right\rangle_{P} \cdot \mathrm{d} V=\left\langle\psi_{a}\right\rangle_{P}
$$

Pour apprécier l'incidence de cette restriction, effectuons un développement limité de $\left\langle\psi_{a}\right\rangle_{P}$. au voisinage de $P$ choisi comme origine du système de coordonnées $x_{i}$ du point $P^{\prime}:\left(P\right.$ barycentre de $R, x_{i}$ repère cartésien $)$. Il vient, compte tenu de la procédure de sommation d'Einstein :

$$
\left\langle\psi_{a}\right\rangle_{p}=\left\langle\psi_{a}\right\rangle_{p}+x_{i}\left(\frac{\partial\left\langle\psi_{a}\right\rangle}{\partial x_{i}}\right)_{p}+\frac{x_{i} x_{i}}{2 !}\left(\frac{\partial^{2}\left\langle\psi_{a}\right\rangle}{\partial x_{i} \partial x_{j}}\right)+\ldots
$$

En intégrant sur $R_{0}$ et en prenant la valeur moyenne :

$$
\begin{aligned}
\left\langle\left\langle\psi_{a}\right\rangle_{P}\right\rangle=\left\langle\psi_{a}\right\rangle_{P} & +\left(\frac{\partial\left\langle\psi_{a}\right\rangle_{P}}{\partial x_{i}}\right)_{P} \frac{1}{R_{0}} x_{i} \mathrm{~d} V \\
& +\frac{1}{2 !}\left(\frac{\partial^{2}\left\langle\psi_{a}\right\rangle}{\partial x_{i} \partial x_{j}}\right) \frac{1}{R_{0}} \int_{P} x_{i} x_{j} \mathrm{~d} V+\ldots
\end{aligned}
$$

$x_{i}$ étant mesuré à partir du barycentre $P$ de $R_{0}$ :

$$
\int_{R_{0}} x_{i} \mathrm{~d} V=0 ; \frac{1}{R_{0}} \int_{R_{0}} x_{i} x_{j} \mathrm{~d} V=0\left(l^{2}\right)
$$

et comme $\left\langle\psi_{a}\right\rangle$ variable pour $x_{i}(0, L)$,

$$
\left(\frac{\partial^{2}\left\langle\psi_{a}\right\rangle}{\partial x_{i} \partial x_{j}}\right)_{p}=0\left(\left\langle\psi_{a}\right\rangle_{p} / L^{2}\right)
$$

En négligeant les termes d'ordre supérieur à 2, on a donc :

$$
\left\langle\left\langle\psi_{a}\right\rangle_{P^{\prime}}=\left\langle\psi_{a}\right\rangle_{P}+0\left(\left\langle\psi_{a}\right\rangle_{P}\left(\frac{l}{L}\right)^{2}\right)\right.
$$

et la deuxième restriction sera satisfaite dès lors que $l \ll L$.

Le changement d'échelle permettant d'assimiler le milieu poreux à un milieu continu fictif équivalent, dans lequel les différentes grandeurs seront définies en moyenne dans cette nouvelle échelle sera donc caractérisé par un V.E.R. dont la dimension, $l$, satisfera à la double restriction : $d<l<L$.

Lorsque $\psi_{a}=\gamma_{a}\left(x_{i}, t\right)$ avec

$$
\begin{aligned}
& \gamma_{\alpha}=\begin{array}{l}
1 \text { dans l'espace des pores } \\
0 \text { dans le solide. }
\end{array} \\
& \frac{1}{R_{0}} \int_{R_{0}} \gamma_{\alpha}\left(x_{i}, t\right) \mathrm{d} V=\varepsilon(P, t)
\end{aligned}
$$

représente la porosité locale du milieu poreux $\varepsilon(\vec{x}, t)$. Pour un milieu poreux indéformable et homogène $\varepsilon=$ cte.

Un raisonnement analogue appliqué à la caractérisation des moyennes surfacique $\bar{\psi}_{a}$ permettrait de montrer qu'en milieu poreux homogène et isotrope $\left\langle\psi_{a}\right\rangle=\bar{\psi}_{a}$ : moyennes surfaciques et volumiques sont équivalentes [3].

\subsection{Relations entre grandeurs macroscopiques et mi- croscopiques}

Les lois qui régissent les phénomènes à l'échelle microscopique, dans les milieux poreux, sont généralement décrites mathématiquement par des équations aux dérivées partielles. C'est le cas pour l'équation de conservation de la quantité de mouvement : équation de Navier, pour l'équation de conservation de l'énergie : équation de Fourier, et pour l'équation de conservation de la masse.

Lorsque l'on cherche à établir les lois macroscopiques locales par des prises de moyennes sur les équations microscopiques, on est alors conduit à utiliser des relations entre les dérivées des grandeurs macroscopiques et celles des grandeurs microscopiques correspondantes. Etant donné que dans la plupart des cas, les grandeurs microscopiques qui caractérisent un phénomène en milieu poreux ne sont pas continues, c'est au sens des distributions que doivent être appliquées les formules de dérivation des grandeurs moyennes sur le V.E.R. Pour la fonction $\psi_{a}$ continuement différentiable dans la phase $\alpha$ mais discontinue sur l'interface $A_{a_{i}}$ dans le milieu poreux, les opérateurs de dérivation s'écrivent alors en valeur moyenne :

$$
\begin{gathered}
\left\langle\nabla \psi_{a}\right\rangle=\nabla\left\langle\psi_{a}\right\rangle+\frac{1}{R_{0}} \int_{A_{a_{i}}}\left|\psi_{a}\right| \vec{n}_{a} \mathrm{~d} A \\
\nabla=\vec{i} \frac{\partial}{\partial x_{1}}+\vec{j} \frac{\partial}{\partial x_{2}}+\vec{k} \frac{\partial}{\partial x_{3}} \\
\left\langle\frac{\partial \psi_{a}}{\partial t}\right\rangle=\frac{\partial}{\partial t}\left\langle\psi_{a}\right\rangle-\frac{1}{R_{0}} \int_{A_{a_{i}}}\left|\psi_{a}\right| \vec{W} \cdot \vec{n}_{a} \mathrm{~d} A
\end{gathered}
$$

$\vec{n}_{\alpha}$ étant la normale à l'interface $A_{a_{i}}$ dirigée vers l'extérieur de la phase $\alpha, \vec{W}$ le vecteur vitesse de déplacement de l'interface $A_{a_{i}}$ et $\left|\psi_{a}\right|$ le saut de $\psi_{a}$ à la traversée de $A_{a_{i}}\left(A_{a_{i}}\right.$ surface séparant les phases $\alpha$ et $i$ ).

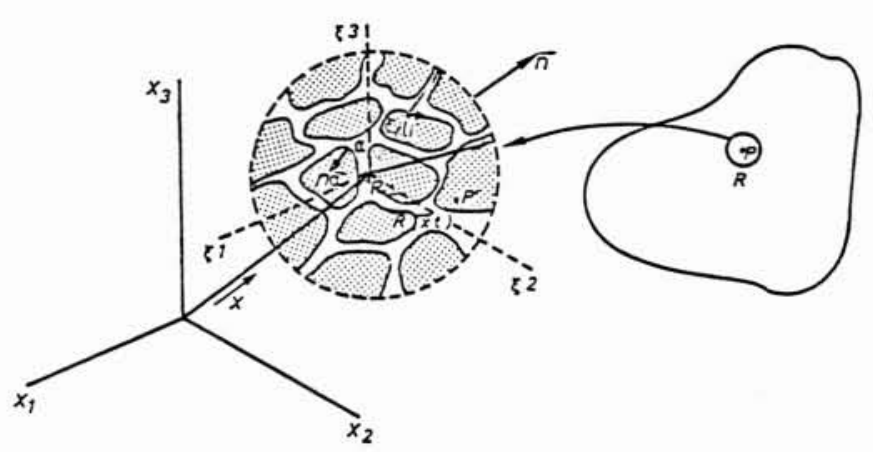

Figure 1

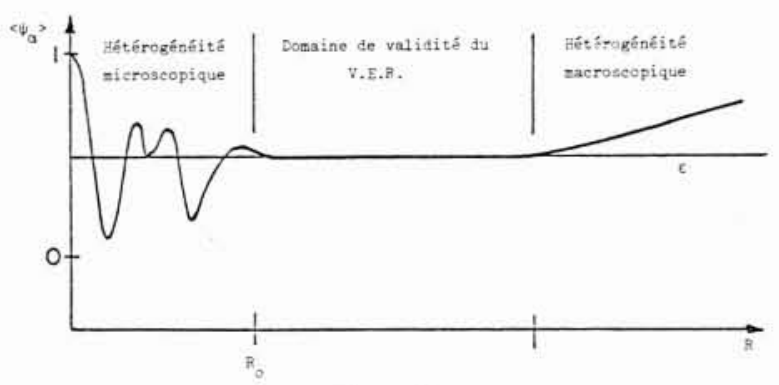

Figure 2 
- Définitions complémentaires :

La moyenne de phase $\alpha$ dans $R_{0}$ étant définie par :

$$
\left.\left.\left\langle\psi_{a}\right\rangle \overrightarrow{(x}, t\right)=\frac{1}{R_{0}} \int_{R_{0}} \psi_{a} \overrightarrow{(x}+\vec{\xi}, t\right) \mathrm{d} V_{\xi}
$$

Nous utiliserons par ailleurs :

- la moyenne intrinsèque de phase:

$$
\left\langle\psi_{a}\right\rangle^{a}(\vec{x}, t)=\frac{1}{R_{\alpha}(x, t)} \int_{R_{a}(\vec{x}, t)} \psi_{a}(\vec{x}+\vec{\xi}, t) \mathrm{d} V_{\xi}
$$

où $R_{a}=$ volume de $\alpha$ dans $R_{0}$.

Etant donné que $\psi_{\alpha}$ est une propriété spécifique à la phase $\alpha$ donc nulle dans les autres phases, la relation entre moyenne de phase et moyenne intrinsèque de phase s'écrit :

$$
\left.\left.\left\langle\psi_{a}\right\rangle \overrightarrow{(x}, t\right)=\varepsilon_{a}(\vec{x}, t)\left\langle\psi_{a}\right\rangle^{a} \overrightarrow{(x}, t\right)
$$

avec

$$
\left.\varepsilon_{\alpha} \overrightarrow{(x}, t\right)=\frac{\left.R_{a} \overrightarrow{(x}, t\right)}{R_{0}}
$$

où $\varepsilon_{\alpha}=\varepsilon S_{\alpha}, \quad S_{\alpha}=$ saturation en phase $\alpha$.

Ces définitions associées à celles relatives à la caractérisation du V.E.R. conduisant aux relations suivantes :

$$
\left\langle\left\langle\psi_{a}\right)^{\alpha}\right\rangle^{a}=\left\langle\psi_{\alpha}\right\rangle^{a} ;\left\langle\left\langle\psi_{a}\right\rangle\right\rangle^{a}=\left\langle\psi_{a}\right\rangle
$$

- la déviation par rapport à la moyenne:

Etant donné que dans le V.E.R. les valeurs de $\psi_{a}$ varient localement d'un point à un autre de la phase $\alpha$ nous noterons par: $\tilde{\psi}_{a}=\psi_{a}-\left\langle\psi_{a}\right\rangle^{a}$ la déviation de $\psi_{a}$. Compte tenu des relations précédentes $\left\langle\tilde{\psi}_{a}\right\rangle^{\alpha}=0$ et la valeur moyenne du produit de deux fonctions $\psi_{1_{a}}$ et $\psi_{2_{a}}$ avec :

$$
\psi_{1 a}=\left\langle\psi_{1_{\alpha}}\right\rangle^{\alpha}+\tilde{\psi}_{a} \text { et } \psi_{2_{a}}=\left\langle\psi_{2_{\alpha}}\right\rangle^{\alpha}+\tilde{\psi}_{2 \alpha}
$$

sera exprimé par :

$$
\left\langle\psi_{1_{a}} \psi_{2_{a}}\right\rangle^{a}=\left\langle\psi_{1_{a}}\right\rangle^{a}\left\langle\psi_{2_{a}}\right\rangle^{a}+\left\langle\tilde{\psi}_{1_{a}} \tilde{\psi}_{2_{a}}\right)^{a}
$$

\section{Equations de bilans microscopiques et macroscopiques}

Si l'on appelle $\psi_{a}$ la valeur spécifique (par unité de masse) d'une propriété extensive $S$ de la phase $\alpha$ et $\rho_{a}$ la masse volumique de $\alpha$ :

$$
\psi_{\alpha}=\frac{\mathrm{d} S}{\mathrm{~d} m_{a}} \quad \rho_{a} \psi_{a}=\frac{\mathrm{d} S}{\mathrm{~d} R_{\alpha}}
$$

$m_{\alpha}$ étant la masse de la phase $\alpha$, l'équation générale de bilan de $S$ à l'échelle microscopique est donnée par l'équation :

$$
\frac{\partial}{\partial t}\left(\rho_{a} \psi_{a}\right)=-\nabla \cdot\left[\rho_{a} \psi_{a} \vec{V}_{\alpha}+\vec{J}\left(\psi_{a}\right)\right]+I_{a}
$$

$\vec{V}_{\alpha}$ étant la vitesse locale de la phase $\alpha$,

$\vec{J}\left(\psi_{a}\right)=\rho_{a} \psi_{a}\left(\vec{V}-\vec{V}_{a}\right)$ représente la densité de flux de diffusion de $\alpha$

$I_{\alpha}$ le taux de production volumique de $S$ dans la phase $\alpha$. En valeur moyenne sur le V.E.R., cette équation conduit à :

$$
\left\langle\frac{\partial}{\partial t}\left(\rho_{a} \psi_{\alpha}\right)\right\rangle=-\left\langle\nabla \cdot\left[\left(\rho_{\alpha} \psi_{\alpha}\right) \vec{V}_{\alpha}+\vec{J}\left(\psi_{\alpha}\right)\right]\right\rangle+\left\langle I_{\alpha}\right\rangle
$$

soit en utilisant les relations de définition et les règles de prises de moyenne relatives aux opérateurs de dérivation : $\frac{\partial}{\partial t} \varepsilon_{\alpha}\left\langle\rho_{a} \psi_{a}\right\rangle^{a}=$

$$
\begin{aligned}
& \left.=-\nabla \cdot\left[\varepsilon_{\alpha}\left\langle\rho_{a} \psi_{a}\right)^{a}\left\langle\vec{V}_{a}\right\rangle^{a}+\varepsilon_{a}\left\langle\left(\rho_{a} \tilde{\psi}_{a}\right) \overrightarrow{\widetilde{V}}_{a}\right\rangle^{a}+\vec{J}\left(\psi_{a}\right)\right\rangle^{a}\right] \\
& \frac{1}{R_{0}} \int_{A_{\alpha_{i}}}\left[\rho_{\alpha} \psi_{\alpha}\left(\vec{V}_{\alpha}-\vec{W}_{a_{i}}\right)+\vec{I}\left(\psi_{a}\right)\right] \cdot \vec{n}_{\alpha} \mathrm{d} A+\varepsilon_{a}\left\langle I_{\alpha}\right\rangle^{a}
\end{aligned}
$$

Cette équation qui représente le bilan macroscopique de $\psi_{a}$ en chaque macropoint $P \in$ (V.E.R.) permet dès lors de traiter le transfert de $\psi_{a}$ dans le milieu poreux comme s'il s'agissait d'un milieu continu.

On notera que par rapport à l'équation microscopique, le bilan ainsi établi à l'échelle du V.E.R. fait apparaître deux termes supplémentaires :

$-\varepsilon_{a}\left\langle\rho_{\alpha} \tilde{\psi}_{a} \overrightarrow{\widetilde{V}}_{a}\right)^{\alpha}$ qui représente une densité de flux de dispersion due aux variations locales de $\vec{V}_{a}$ et $\rho_{a} \psi_{a}$,

$$
-\frac{1}{R_{0}} \int_{A_{a_{i}}}\left[\rho_{\alpha} \psi_{a}\left(\vec{V}_{\alpha}-\vec{W}_{a_{i}}\right)+\vec{J}\left(\psi_{a}\right)\right] \cdot \vec{n}_{a} \mathrm{~d} A
$$

qui représente le transfert de $\psi_{a}$ à travers les interfaces $A_{a_{i}}$ situés dans le V.E.R. Ce terme ne s'identifie à zéro que si $A_{a_{i}}$ est une surface matérielle et s'il n'y a pas de transfert entre phases.

Les solutions relatives aux variables dépendantes $\left\langle\rho_{a} \psi_{a}\right\rangle^{a}$ et $\varepsilon_{a}$ pour chacune des phases, pourraient en principe être obtenues à partir des équations précédentes dès lors que les conditions initiales et aux limites exprimées à partir des mêmes variables macroscopiques seraient connues. Il convient de noter toutefois que même dans une telle hypothèse, il faudrait connaître de plus, non seulement

$$
\left.\left.\vec{V}_{a}\right\rangle^{a}, \overrightarrow{\langle J}\left(\psi_{a}\right)\right\rangle^{\alpha},\left\langle I_{\alpha}\right\rangle^{a},
$$

mais également les densités de flux qui transfèrent à travers les interfaces ainsi que les densités de flux de dispersion. Dans l'état actuel des connaissances et des moyens expérimentaux disponibles, ces déterminations sont impossibles dans le cas général.

Diverses tentatives ont néanmoins été effectuées dans le cas le plus simple de l'écoulement de fluides monophasiques en vue de justifier théoriquement la loi expérimentale de Darcy. Si dans l'ensemble, elles aboutissent correctement, ceci ne peut toutefois se faire que par la prise en compte d'hypothèses complémentaires permettant :

- d'exprimer les intégrales de surface relatives à l'influence du champ de pression microscopique et le frottement pariétal sur les surfaces solides,

- de négliger l'influence des termes de dispersion.

Nous ne nous étendrons pas davantage sur ce problème de changement d'échelle dont on trouvera une analyse beaucoup plus détaillée dans les références [1], [2], [3], [4] et [5] notamment. 


\section{Modélisation mathématique des transferts de chaleur et de masse}

Dans la suite de cette analyse, nous nous limiterons exclusivement au problème du transfert de chaleur et de masse dans des milieux poreux finement divisés, homogènes indéformables saturés par un constituant fluide non réactif éventuellement sous ces deux phases. La température de la phase fluide sera en outre supposée comprise entre les deux valeurs limites correspondant aux transitions liquide-solide et liquide-vapeur, les processus de congélation et d'autovaporisation n'étant pas examinés ici.

Les vitesses d'écoulement des phases seront par ailleurs considérées suffisamment faibles pour que les interactions dynamiques, les effets de dissipation et de compressibilité soient négligeables.

Compte tenu de cet ensemble d'hypothèses et des restrictions spécifiques à la mise en œuvre de la procédure de changement d'échelle, les équations macroscopiques de conservation de la masse, de la quantité de mouvement et de l'énergie déduites des équations microscopiques s'écrivent en supprimant les ( ) et en affectant les indices $o$ à la phase solide et $\alpha$ (égal à $l$ pour le liquide et à $g$ pour le gas) aux phases fluides.

$$
\begin{aligned}
& \quad \frac{\partial}{\partial t}\left(\varepsilon \rho_{a} S_{a}\right)=-\nabla \cdot\left(\vec{J}_{a_{e}}+\vec{J}_{a_{d}}\right)+I_{a} \\
& \cdot \vec{J}_{a_{e}}=-\frac{\overline{\bar{K}}_{a}}{v_{a}} \nabla \cdot\left(P_{a}-\rho_{a} \vec{g} z\right) \\
& \cdot \rho_{o}(1-\varepsilon) \frac{\partial h_{a}}{\partial t}+\sum_{a} \rho_{a} \varepsilon S_{a} \frac{\partial h_{a}}{\partial t}= \\
& \left.\quad=-\nabla \cdot \vec{J}_{q}-\sum_{a} \vec{J}_{a_{c}}+\vec{J}_{a_{d}}\right) \cdot \nabla h_{a}-\sum_{a} I_{a} h_{a}+\Phi \\
& \cdot \sum_{a} I_{a}=0 ; \sum_{a} S_{a}=1 ; \quad P_{g}-P_{l}=P_{c}\left(S_{a}, T\right)
\end{aligned}
$$

$\rho_{a}, v_{a}, h_{a}, P_{a}$ représentent respectivement la masse volumique, la viscosité cinématique, l'enthalpie massique et la pression de la phase $\alpha, S_{\alpha}$ la saturation en phase $\alpha$ (fraction du volume des pores occupés par le liquide et la vapeur), $\vec{J}_{a_{c}}$ et $\vec{J}_{a_{d}}$ les densités de flux de masse transportées par convection et diffusion, $I_{a}$ les termes traduisant les changements de phase (évaporation-condensation), $\overline{\bar{K}}_{\alpha}$ le tenseur de perméabilité à la phase $\alpha, \vec{J}_{q}$ la densité de flux thermique associée aux transferts par conduction, $P_{c}$ la pression capillaire d'équilibre entre phases et $\Phi$ un terme source (rayonnement par exemple).

La fermeture de ce système peut être effectuée grâce à la connaissance des lois constitutives justifiées par J.C. Bennet en 1981, des équations d'équilibre et d'état des différentes phases et de certains coefficients phénoménologiques. Les lois constitutives concernent respectivement :

- le transfert de chaleur associé à la diffusion thermique dont on peut montrer qu'il peut se mettre sous la forme :

$$
\vec{J}_{q}=-\overline{\bar{\lambda}}^{*} \cdot \nabla T
$$

$$
\begin{aligned}
& \text { avec : } \\
& \begin{aligned}
\overline{\bar{\lambda}}^{*}=\left[(1-\varepsilon) \lambda_{o}\right. & \left.+\sum_{a} \varepsilon S_{a} \lambda_{a}\right] \overline{\bar{I}}+\left(\lambda_{a}-\lambda_{l}\right) \overline{\bar{K}}_{o} \\
& +\left(\lambda_{1}-\lambda_{g}\right) \overline{\bar{K}}_{\mathrm{c}}+\left(\lambda_{g}-\lambda_{o}\right) \overline{\bar{K}}_{g}+\overline{\bar{K}}_{D}
\end{aligned}
\end{aligned}
$$

où $\overline{\bar{K}}_{a}$ et $\overline{\bar{K}}_{b}$ représentent des tenseurs du second ordre que nous expliciterons ultérieurement, $\overline{\bar{I}}$ le tenseur unité, $\lambda_{a}$, la conductivité thermique de chacune des phases homogène.

- le transfert de matière associé à la diffusion moléculaire dont on peut montrer également qu'il peut se mettre sous la forme :

$$
\left.\vec{J}_{a_{D}}=-\overline{\bar{D}} \cdot \nabla \cdot \rho_{a_{i}}: \sum \rho_{a_{i}}=\rho_{a} ; \overline{\bar{D}}=\mathscr{( \overline { I }}+\overline{\bar{J}}\right)
$$

l'indice, $i$, représentant les différentes espèces chimiques constituant la phase $\alpha, \overline{\bar{D}} *$ le tenseur de diffusion massique équivalent, $\mathscr{D}$ le coefficient de diffusion moléculaire de l'espèce $i$ dans la phase homogène $\alpha$ et $\bar{J}_{a}$ un tenseur du second ordre traduisant l'influence de la tortuosité du milieu poreux.

Les équations d'état, dans le cadre des hypothèses retenues, concernent quant à elles : $\rho_{a}(T) ; \sigma(T) ; \mu_{a}(T)$ et $C_{a}=\partial h_{a} / \partial T$ où $\sigma$ est la tension interfaciale, $\mu_{a}$ la viscosité dynamique et $C_{a}$ la chaleur spécifique de la phase $\alpha$.

Dans l'état actuel des connaissances, les relations permettant d'expliciter $P_{c}\left(T, S_{\alpha}\right)$ et les tenseurs de perméabilité $\overline{\bar{K}}_{a}$, de conductivité thermique équivalente $\overline{\bar{\lambda}}^{*}$ et de diffusion massique $\overline{\bar{D}}^{*}$ ne peuvent être obtenues que par voie expérimentale. Dépendant de la structure géométrique intime du milieu poreux, la plupart de ces grandeurs s'explicitent en effet sous la forme d'intégrales de surface dont la détermination est pour l'instant hors de portée.

Pour nous limiter au seul problème thermique dans lequel les effets diffusifs sont caractérisés par le tenseur de conductivité apparente $\bar{\lambda}^{*}$, on notera en effet qu'à l'exception de la grandeur scalaire $(1-\varepsilon) \lambda_{0}+\sum \varepsilon S_{a} \lambda_{a}$ aisément accessible, tous les termes complémentaires :

$$
\begin{aligned}
\overline{\bar{K}}_{o} & =\frac{1}{R_{0}} \int_{A_{o l}}|T| n_{o l} \mathrm{~d} A_{o l} ; \\
\bar{K}_{l} & =\frac{1}{R_{0}} \int_{A_{l_{g}}}|T| n_{l g} \mathrm{~d} A_{l g} ; \\
\overline{\bar{K}}_{v} & =\frac{1}{R_{0}} \int_{A_{g o}}|T| n_{g o} \mathrm{~d} A_{k_{o}} ;
\end{aligned}
$$

traduisant l'influence de la distribution des phases exigent la connaissance des aires interfaciales.

Déjà complexe dans le cas de la conduction thermique seule, la prédétermination de ce tenseur l'est encore plus lorsque les phases fluides sont en écoulement par suite de l'intervention des effets de dispersion venant se superposer au champ convectif moyen. Généraleme:It identifiée à une loi de type gradient, la densité de flux thermique associé aux effets dispersifs est telle que :

$$
\overline{\bar{K}}_{D} \cdot \nabla T \approx \sum_{a} \rho_{a} \varepsilon S_{a} C_{a}(\tilde{T} \overrightarrow{\tilde{V}})
$$




\section{Exemples d'applications : \\ convection naturelle en milieu poreux saturé par une phase fluide unique transferts couplés de chaleur et de masse en milieu poreux non saturé}

Afin d'illustrer à travers quelques exemples l'adéquation entre modélisation mathématique et phénomènes physiques, deux résultats de validation des modèles théoriques seront présentés. Le premier concerne la convection naturelle d'origine thermique, le second la thermomigration i.e. les transferts couplés de chaleur et de masse en milieu poreux non saturé. Dans les deux cas, les vitesses des fluides et les écarts de température sont suffisamment faibles pour que les écoulements puissent être considérés comme darcéens et les effets de dispersion négligeables. Les milieux poreux sont en outre non seulement homogènes, mais également isotropes afin que la détermination des perméabilités, conductivités thermiques et diffusivités massiques apparentes puissent s'effectuer sans de trop grandes difficultés. Cet ensemble de conditions permettant de se situer dans un contexte ou d'une part la modélisation peut être considérée comme a priori satisfaisante et ou, d'autre part, les coefficients intervenant dans les équations constitutives peuvent être déterminés au moyen d'expériences spécifiques, il s'ensuit que la comparaison entre simulations physiques et résultats théoriques constitue le critère d'appréciation de la validité des modèles.

\subsection{Convection naturelle en milieu poreux}

Ce sujet a donné lieu à de très nombreuses études au cours des deux dernières décennies, notamment dans le cas de couches poreuses homogènes isotropes de grande extension [6], [7]. Pour cette configuration, les résultats acquis tant en ce qui concerne les critères d'apparition de la convection, que la forme des écoulements ou le transfert de chaleur moyen induit, ont non seulement permis d'apprécier la validité des modèles, mais également d'identifier certaines limites de validité. Aujourd'hui bien connus et par ailleurs très largement diffusés, ces résultats ne seront pas rappelés ici.

Pour illustrer la comparaison entre expérience et calcul, nous présenterons plutôt des résultats récents concernant l'influence du confinement latéral et des conditions aux limites thermiques sur la convection naturelle dans une cavité cylindrique de section droite circulaire d'axe vertical contenant un milieu poreux saturé par un fluide de Boussinesq (Fig. 3). Cette cavité, réalisée dans un matériau solide de conductivité thermique $\lambda_{p}$ a une hauteur $H$ et un diamètre $D$. La paroi latérale a une épaisseur, $e$, et les plans horizontaux situés à $z^{\prime}=0$ et $z^{\prime}=H$ sont imperméables et isothermes maintenus en régime permanent aux températures $T_{2}$ et $T_{1}$ constantes avec $T_{2}=T_{1}+\Delta T$, [8].

Contrairement à ce qui se passe pour la couche poreuse de grande extension latérale, les phénomènes ne dépendent plus seulement ici du seul nombre de Rayleigh :

$$
R a_{H}^{*}=g \frac{\gamma\left(\rho_{f} C_{f}\right)}{v} \frac{K}{\lambda^{*}} H \cdot \Delta T
$$

( $\gamma$ étant le coefficient d'expansion thermique du fluide) mais aussi des rapports : $\Lambda=\lambda^{*} / \lambda_{p} ; R_{0}=H / D$ et $E=2 e / D$

L'étude théorique de ce problème effectuée à l'aide du système d'équations présenté dans le paragraphe précédent a permis d'obtenir de nombreux résultats originaux bien vérifiés expérimentalement [9]. Nous mentionnerons notamment :

- la mise en évidence de l'effet stabilisateur créé par le confinement géométrique (l'accroissement du rapport $H / D$ retarde l'apparition de la convection), (Fig. 4);

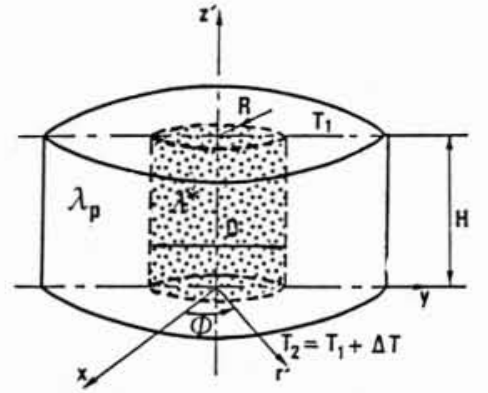

Figure 3

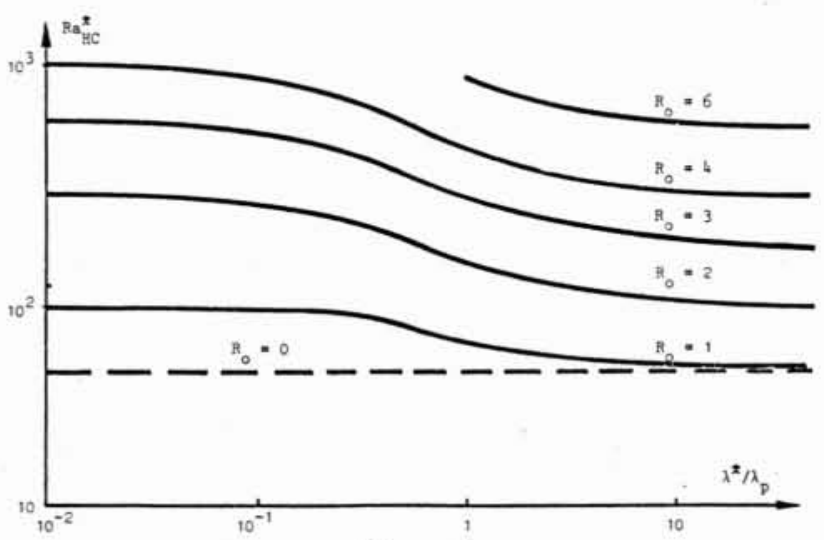

Figure 4 
- l'existence d'une valeur critique du rapport $H / D$, valeur au-delà de laquelle la naissance de la convection est exclusivement contrôlée par le diamètre de la cavité. Le nombre de Rayleigh construit avec cette grandeur, $R a_{D}^{*}$ toutes choses étant égales par ailleurs, est alors constant (Fig. 5);

- la mise en évidence de l'effet stabilisateur créé par la nature du matériau constituant la paroi latérale (l'accroissement de la conductivité thermique de la paroi retarde l'apparition dès la convection);

- la configuration générale des écoulements convectifs;

- les relations donnant le transfert de chaleur moyen explicité par le nombre de Nusselt en fonction des différents paramètres. Les comparaisons expérience-résultats théoriques correspondants sont présentés sur les figures 6 et 7).

\subsection{Transferts de chaleur et de masse dans les milieux poreux non saturés}

Malgré une importance pratique considérable dans divers domaines techniques ou situations naturelles, ce problème a été relativement peu étudié. Les difficultés liées à l'expérimentation et en particulier à la caractérisation des coefficients de transferts (masse et chaleur) constituent il est vrai un ensemble d'éléments assez dissuasifs.

En dépit de ces difficultés, un travail de recherche, constituant selon nous la mise à l'épreuve la plus compléte du système d'équations régissant les phénomènes $a$, néanmoins, été effectué au cours de ces toutes dernières années [11], [12].

Le problème traité concerne les transferts couplés de chaleur et de masse au sein d'un milieu poreux homogène isotrope indéformable non saturé en eau, soumis à de faibles gradients thermiques, dans un domaine de température compris entre $0^{\circ} \mathrm{C}$ et $100^{\circ} \mathrm{C}$. La pression totale de la phase gazeuse étant uniforme et constante, les transferts de masse sont respectivement associés aux gradients de pression de vapeur et de pression capillaire tandis que les transferts d'énergie s'effectuent principalement par diffusion thermique et par transfert de chaleur latente due aux effets de vaporisation-condensation.

Dans le cadre des hypothèses retenues, les densités de flux associés à ces différents transferts s'écrivent respectivement :

$$
\overrightarrow{J_{l}}=-\rho_{l}\left(D_{\theta_{l}} \nabla \theta_{l}+D_{T_{l}} \nabla T\right)+\rho_{l} K_{l} \vec{k}
$$

pour la densité de flux de masse en phase liquide,

$$
\vec{J}_{v}=-\rho_{l}\left(D_{\theta_{v}} \nabla \theta_{l}+D_{T_{v}} \nabla T\right)
$$

pour la densité de flux de masse en phase vapeur, et

$$
\vec{J}_{q}=-\lambda^{*} \nabla T+\vec{J}_{t} h_{l}+\vec{J}_{v} \mathscr{S}
$$

pour la densité de flux de chaleur (avec $\vec{J}_{1}=\vec{J}_{1}+\vec{J}_{v}$ densité de flux de masse totale, $\mathscr{f}$ chaleur latente de changement de phase.).

Les coefficient de transfert de masse isotherme $D_{\theta_{1}}, D_{\theta_{v}}$, non isothermes $D_{T_{l}}, D_{T_{\mathrm{r}}}$ et la conductivité thermique équivalente $\lambda^{*}$ sont des fonctions compliquées de la saturation en eau et de la température. En raison des couplages existants entre transferts de masse et transfert de chaleur, on notera par ailleurs les difficultés de mesure de la conductivité thermique équivalente $\lambda^{*}$ en dehors de toute influence du transfert de matière.

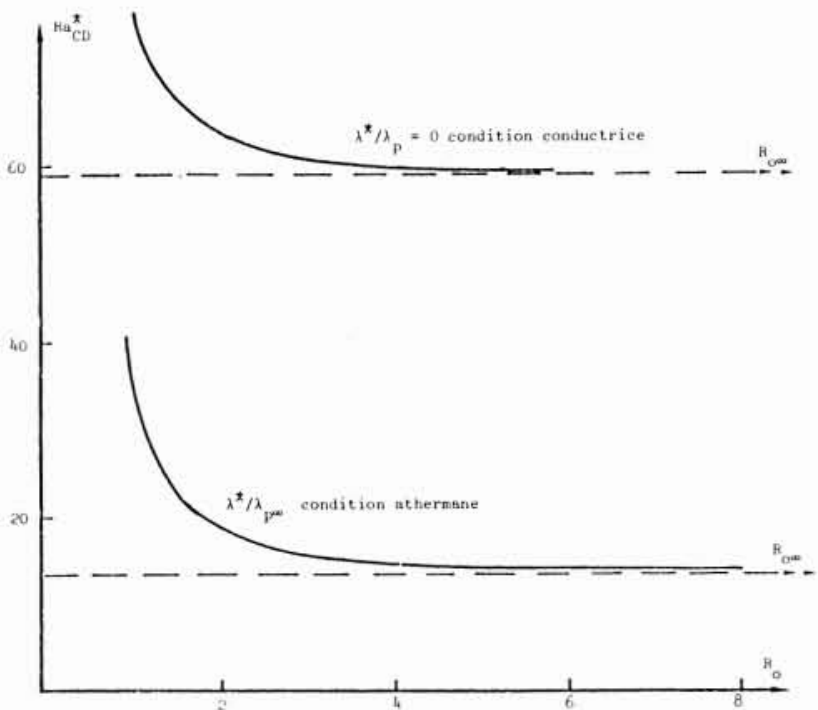

Figure 5
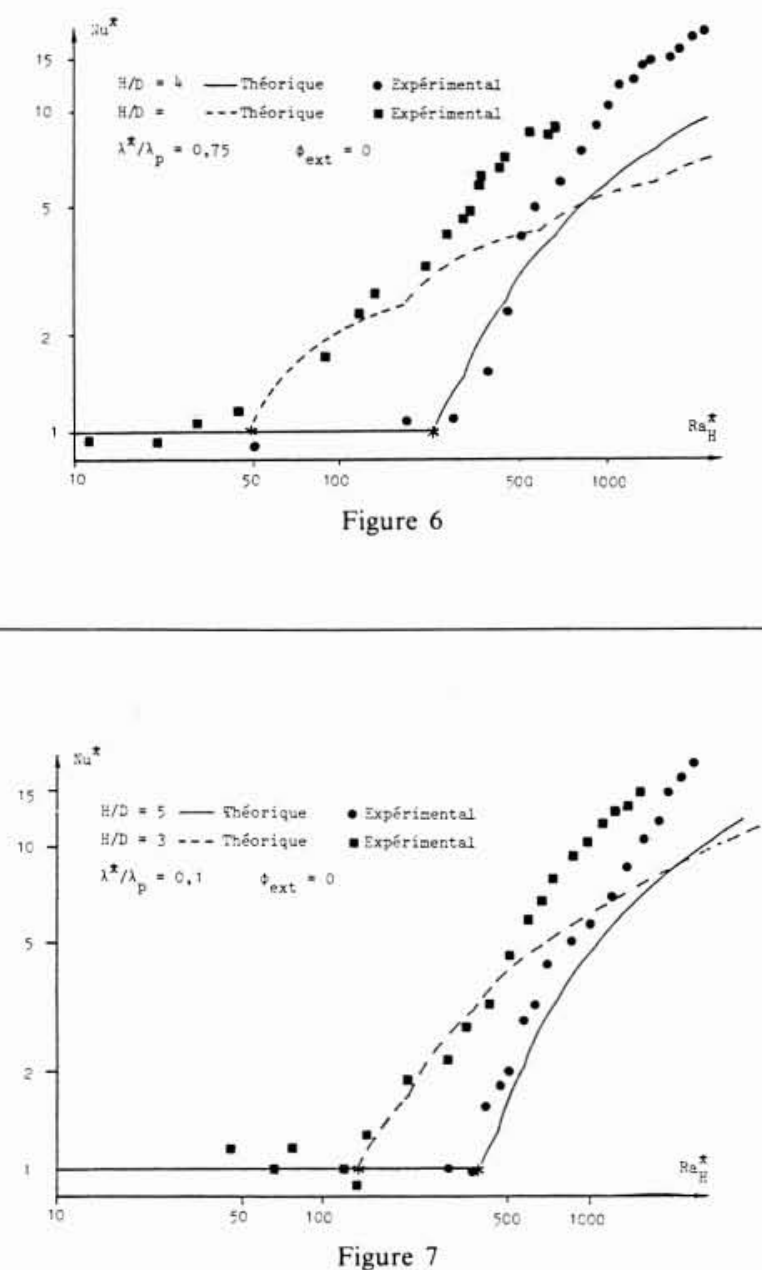

Figure 7 


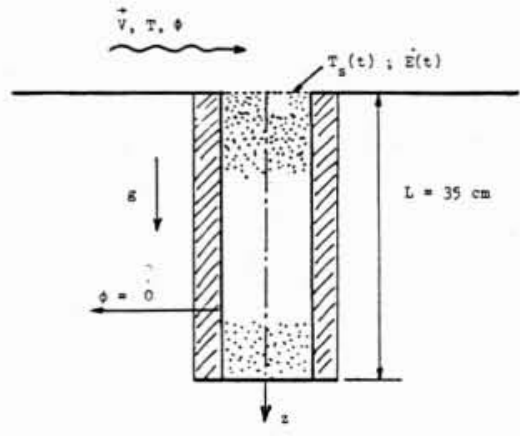

Figure 8

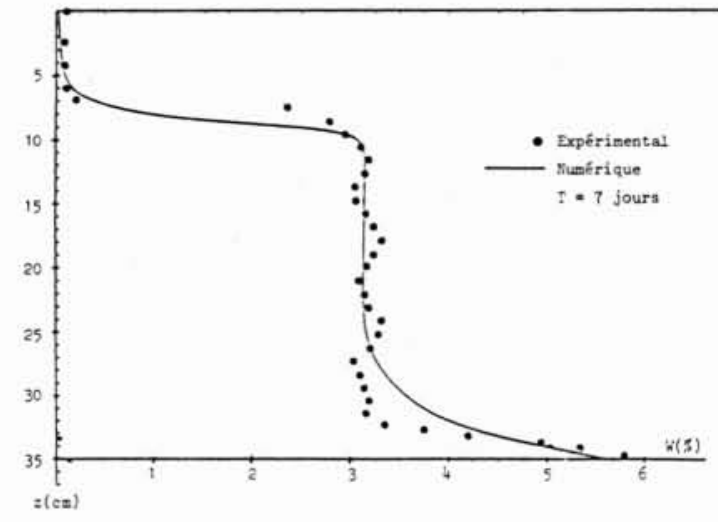

Figure 9

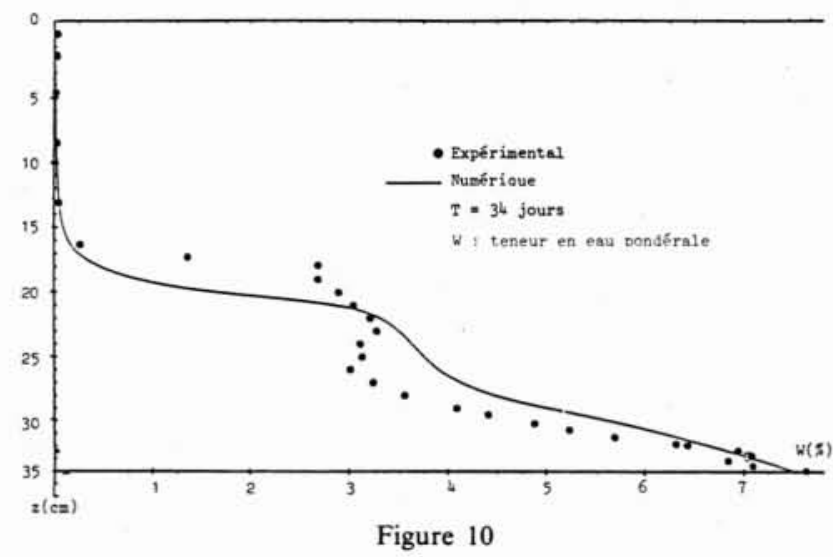

Figure 10

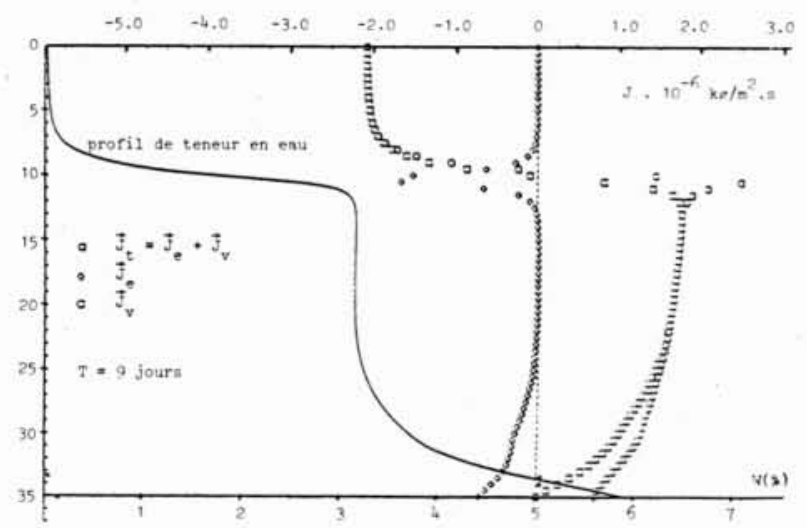

Figure 11
Après avoir déterminé les différents coefficients intervenant dans les lois constitutives, au moyen d'expériences spécifiques [12], le test de validation du modèle mathématique a été effectué par comparaison des profils expérimentaux et théoriques de température et de teneur en eau se développant dans un processus d'évaporation-condensation unidirectionnel.

La configuration physique retenue est représentée sur la figure 8 . Elle correspond à un milieu poreux mis en contact à la partie supérieure avec un écoulement d'air à température, vitesse et humidité relative contrôlées et à la partie inférieure avec une surface imperméable isotherme à une température inférieure à celle de l'air s'écoulant en surface. La compétition qui s'établit dans cette expérience entre l'évaporation superficielle et les mécanismes de diffusion de masse interne conduit à l'apparition de profils de teneur en eau tout à fait remarquables permettant une comparaison significative des résultats. Cette comparaison est effectuée sur les figures 9 et 10 , montrant à l'évidence le caractère tout à fait représentatif de la physique des phénomènes, du modèle de transferts couplés. La figure 11 indique clairement, quant à elle, comment s'établit le partage des flux massiques en phase vapeur au voisinage du front d'évaporation. 


\section{Conclusion}

Les exemples que nous avons choisis pour illustrer l'adéquation entre modélisation mathématique et phénomènes physiques en milieux poreux finement divisés satisfaisant au critère d'homogénéité macroscopique, ne constituent qu'un ensemble très restreint des classes de problèmes pour lesquels cette adéquation a été confirmée de façon tout à fait satisfaisante.

Bien que de tels modèles puissent donc être utilisés dès à présent en vue d'améliorer notre compréhension de certains phénomènes naturels ou pour servir de guide dans la définition des conditions optimales d'exploitation thermique du sous-sol, c'est néanmoins avec prudence que l'utilisation des résultats issus de ces modélisations doit être envisagée.
Indépendamment des problèmes posés par la définition précise des dimensions, forme géométrique et conditions aux limites des gisements exploités, il convient de noter en effet d'une part que les structures géologiques sont généralement hétérogènes, et d'autre part que les coefficients thermophysiques, caractérisant l'aptitude aux transferts des milieux poreux en général, restent des fonctions mal connues des propriétés aussi bien structurales que physiques de ces milieux.

Si les efforts de recherche engagés actuellement sur les problèmes de changement d'échelle, laissent entrevoir des possibilités d'étude raisonnable des milieux hétérogènes, beaucoup reste encore à faire avant de pouvoir accéder à la connaissance des lois auxquelles obéissent les paramètres thermophysiques.

\section{Bibliographie sommaire}

[1] Marle. - Ecoulements monophasiques en milieu poreux. Revue Institut Français du Pétrole, 22, 10, 1471-1509 (1967).

[2] WhitAKer. - Advances in theory of fluid motion in porous media. Industrial and Engineering Chemistry, 61, 12, pp. $15-26$ (1969).

[3] Whitaker. Simultaneous heat, mass and momentum transfer in porous media. A theory of drying. Advances in Heat Transfer, Vol. 13, pp. 119-203 (1977)

[4] Gray, O'Nell. - On the general equations for flow in porous media and their reduction to Darcy's law. Water Resources Research, 12, pp. 148-154 (1976).

[5] MARLE. - From the pore scale to the macroscopic scale : equations governing multiphase fluid flow through porous media flow and transport in porous media. Edited by Verruijt and Barrenus - A.A. Belkema/Rotterdam, pp. 57-61 (1981).

[6] Combarnous, Bories. - Hydrothermal convection in saturated porous media. Advances in Hydroscience 10, pp. 231-307, Academic Press New-York (1975).
[7] Combarnous. - Convection naturelle en milieu poreux et systèmes géothermiques. Sixième Conférence Internationale sur le Transfert de Chaleur, Toronto, Vol. 7, $1^{\text {te }}$ Partie, pp. 35-49, août 1978.

[8] Bories, Deltour. - Influence des conditions aux limites sur la convection naturelle dans un volumè poreux cylindrique. Int. J. Heat Mass Transfer, 23, 6, pp. 765-771 (1980)

[9] Deltour, Convection naturelle au sein d'un milieu poreux saturé confiné dans un domaine cylindrique vertical. Influence des conditions à la limite latérale. Thèse de Doctorat d'Etat, I.N.P. Toulouse, 25 octobre 1982.

[10] BenNet. - Contribution à l'étude thermodynamique des milieux poreux non saturés avec changement de phase. Thèse de Doctorat d'Etat, U.S.T.L., Montpellier (1981).

[11] RECAN. - Simulation numérique du comportement thermique et hydrique d'un sol nu. Application à l'étude de l'évaporation ou télédétection. Thèse de Docteur-Ingénieur, I.N.P. Toulouse, 28 mai 1982.

[12] Crausse. - Etude fondamentale des transferts couplés de chaleur et d'humidité en milieu poreux non saturé. Thèse de Doctorat d'Etat, I.N.P. Toulouse, 17 janvier 1983. 
L'ensemble des discussions qui se sont développées à la suite des divers exposés de cette session fait apparaître globalement deux types de préoccupations de la part des intervenants :

- la connaissance précise des paramètres conditionnant les phénomènes de transfert de chaleur et d'humidité dans le sol et en surface,

- l'aptitude des modèles théoriques présentés à prévoir la réalité physique.

Monsieur GOLDSTEIN pose en premier lieu le problème de la détermination des coefficients de diffusion pris en compte au cours de simulations numériques du comportement thermique et hydrique de sols, présentées dans le cadre d'études développées à l'Institut de mécanique des fluides de Toulouse. Monsieur BorIES précise qu'en ce qui concerne la conductivité thermique, ce paramètre a été déterminé à l'aide d'une méthode expérimentale de régimes stationnaires. Les coefficients de diffusion de masse ont été, également, évalués par des expériences spécifiques, en admettant un découplage au niveau de la quantité de mouvement entre les écoulements en phase gazeuse et en phase liquide.

La présentation de relevés de températures dans un bassin sédimentaire effectuée ensuite par Monsieur WATREMEZ, soulève, entre autres, le problème de la précision des mesures in situ par forage : risque de convection autour du point de mesure (Monsieur BORIES), perturbation de la mesure par le forage lui-même (Monsieur BANAL). D'autre part, le champ de température dans le sol peut être également influencé par l'existence d'incidents de structure (batholites) d'après Monsieur GRJEBINE, ou par la présence dans les fractures, de régimes de convection naturelle (Monsieur WATREMEZ) induisant des inversions de gradients de température, soulignées par Monsieur BORIES. Les variations du paramètre conductivité thermique (de 1 à 5 ) utilisé dans les modèles thermiques du Bassin Parisien, paraissent élevées à Monsieur FIRDAOUSS. Monsieur WATREMEZ fait alors remarquer que ces variations, obtenues par des mesures effectuées à faible profondeur ou en laboratoire, sont normales, en raison de la présence de fluides dans les roches qui modifient sensiblement la conductivité thermique équivalente.

La représentativité des modèles théoriques utilisés et des hypothèses formulées, en vue de simuler les champs de température et d'humidité dans les sols, apparaît ensuite comme le thème principal des discussions.

En ce sens, le concept de similitude, introduit dans la modélisation de la variabilité spatiale de paramètres macroscopiques (perméabilité, conductivité, etc.) par l'exposé de Monsieur BRUNET, soulève une question de Monsieur QUINTARD quant à la justification de pratiquer la similitude sur des grandeurs macroscopiques faisant elles-mêmes intervenir des couplages de phénomènes microscopiques ayant des similitudes différentes. Monsieur BRUNET reconnaît que si cette approche implique de fortes hypothèses, elle fournit en général une bonne adéquation entre modèles et mesures, dans le cas de milieux poreux à granulométrie peu étendue (exemple des sols de type "sable "); par contre, lorsque les tailles de particules sont très différentes, la similitude n'est plus, effectivement, aussi bonne. Monsieur GRJEBINE pose le problème important du rôle joué par la couverture biologique dans la modulation des phénomènes de transfert sol-atmosphère, particulièrement lors de l'existence de mécanismes de surface séquentiels, tels que effets de pluie et évaporation de l'humidité, se traduisant par des apports ou des pertes de calories. Monsieur BRUNET souligne que, dans le cas de sols nus, des gradients thermiques très importants peuvent exister dans les couches superficielles à l'échelle de la journée, alors qu'en présence d'un couvert végétal, ils seront en moyenne beaucoup plus modérés. A l'échelle de l'année, la différence est beaucoup moins sensible au niveau du stockage de chaleur, les températures évoluant de manière semblable en profondeur. Il semble donc, comme le fait remarquer Monsieur le Président, que la prise en compte de phénomènes de type biologique dans les modèles prévisionnels de transfert permettrait une approche plus précise de la réalité, en particulier lors des opérations (sensiblement différentes) de séchage de grains de nature biologique ou minérale.

Monsieur BENET, après avoir décrit une modélisation mathématique locale des sols non saturés, basée sur la Thermodynamique des Processus Irréversibles, présente des résulats expérimentaux sur le comportement thermique et hydrique de la couche d'un sol située au-dessus d'un stockage de chaleur. Monsieur GRJEBINE s'interroge à nouveau quant à l'incidence sur ces résultats de la présence en surface d'une couche imperméable à la vapeur. Monsieur BENET répond que dans la configuration expérimentale étudiée, les transferts d'énergie en phase gazeuse, et également en phase liquide sont négligeables. Monsieur BORIES fait alors remarquer qu'après les exposés de MM. BRUNET et BENET on peut finalement s'interroger quant à l'intérêt d'avoir recours à des modèles compliqués, quand on se rend compte que dans les deux cas, on arrive à rendre compte de certaines expériences avec des simplifications draconniennes. Il précise qu'en l'occurence les deux cas présentés ne sauraient avoir valeur de règle générale et qu'il convient de procéder à des analyses de sensibilité aux paramètres avant toute simplification a priori. Des travaux effectués à Toulouse par MM. RECAN et CRAUSSE illustrent d'ailleurs parfaitement les risques auxquels peuvent conduire ces réductions de modèles dans l'étude des transferts couplés en milieu poreux. Monsieur BRUNET souligne qu'on ne peut parler d'un modèle qu'en fonction des objectifs qu'on lui assigne. Monsieur BENET précise alors qu'il faut quand même distinguer les modèles permettant une analyse fine de la physique des phénomènes et des modèles de prévision liés à une application.

Monsieur le Président conclut en développant l'idée que le modèle général est élaboré pour aborder toute situation, puis on le simplifie, compte tenu des variables qui paraissent essentielles dans la configuration physique étudiée.

Nota: Le texte ci-dessus fait la synthèse des discussions qui ont suivi la présentation des quatre communications présentées le 14 novembre 1984 sur " les transferts thermiques dans le sous-sol ". Cette synthèse a été préparée par M. P. Crausse, Maitre de Conférences à l'INP de Toulouse. Pour la commodité des lecteurs, nous la présentons à la suite de chacune des quatre communications concernées. 